\title{
Three-Dimensional Magnetically-Oriented Microcrystal Array: A Large Sample for Neutron Diffraction Analysis
}

\author{
T. Kimura ${ }^{1}$, F. Kimura ${ }^{1}$, K. Matsumoto ${ }^{1}$ and N. Metoki ${ }^{2}$ \\ ${ }^{1}$ Kyoto University \\ 2Japan Atomic Energy Agency \\ Japan
}

\section{Introduction}

Structure determination of biomolecules is of great importance because the structure is closely related to biological functions. Some proteins are activated when binding with ligands; a drug molecule functions by binding to a specific protein site (Sousa et al., 2000; Graves et al., 1994). The structure determination is important not only for biomolecules but also for many inorganic, organic, and polymeric crystals that are key materials in materials and pharmaceutical sciences.

Currently, three major methods to solve the structure of molecules are known: solution nuclear magnetic resonance (NMR) (Herrmann et al., 2002), X-ray and neutron single crystal analysis (Blundell et al., 1976; Kendrew et al., 1958; Blake et al., 1965), and X-ray powder diffraction (Margiolaki et al, 2008; Hariss et al., 2004) methods. The solution NMR method has an advantage over diffraction methods because it does not require crystals. However, it can be applied only to proteins with lower molecular weights. X-ray and neutron singlecrystal analysis is the most powerful method, but it is sometimes difficult to grow a crystal (Ataka et al., 1986) sufficiently large for conventional or synchrotron single-crystal X-ray measurement. The size requirement is much more severe for neutron diffraction measurements (Niimura et al., 1999). The X-ray powder method can be performed if microcrystalline powders are available, but an appropriate refinement of many parameters is needed to obtain a reliable result. Preferential orientation of a powder sample is utilized to produce single-crystal-like diffraction data (Wessels et al., 1999). However, the data quality strongly depends on the quality of the orientation.

It is well known that feeble magnetic materials such as most biological, organic, polymeric and inorganic materials respond to applied magnetic fields, although the response is weak. A number of studies on the magnetic alignment of such materials have been reported (Maret et al., 1985; Asai et al., 2006). The study of the feeble interaction of diamagnetic materials with an applied magnetic field and its application are now recognized as an immerging area of science and technology, and named "Magneto-Science".

We have recently proposed a method that enables to convert a diamagnetic or paramagnetic microcrystalline powder to a "pseudo single crystal (PSC)" (T. Kimura et al. 2005; T. Kimura, 
2009a)(Fig. 1). A PSC is a composite in which microcrystals are oriented three-dimensionally in a resin matrix. A PSC is also referred to as a "three-dimensional magnetically-oriented microcrystal array" (3D-MOMA) (F. Kimura et al., 2011). This method enables us to perform a single-crystal analysis of a material through alignment of its powder sample. The composite is fabricated using magnetic alignment of a microcrystal suspension under an elliptically rotating magnetic field, followed by consolidation of the suspending matrix.

The degree of alignment strongly depends on the diamagnetic anisotropy of the crystal, applied field strength, and size of microcrystals to be aligned. If these conditions are appropriately satisfied, the obtained 3D-MOMA can produce well-separated diffraction spots that allow single-crystal analysis. This method can be applied to biaxial crystals including the triclinic, monoclinic, and orthorhombic systems which have three different magnetic susceptibility values. We only obtain fiber diffraction patterns for uniaxial crystals even if we apply the 3D-MOMA method. This method does not work for the cubic system.

However, the 3D-MOMA method has some drawbacks including the limitation of the suspending medium, the difficulty of recovering a precious sample, and the broadening of diffraction spots by consolidation. A solution to these problems may be measurement of the X-ray diffraction patterns directly from a three-dimensional magnetically-oriented microcrystal suspension (3D-MOMS) without consolidation of the suspending medium. Xray diffraction from magnetically oriented solutions of macromolecular assemblies was reported (Glucksman et al., 1986; Samulski et al., 1986). Also, small-angle X-ray scattering of colloidal platelets (van der Beek et al., 2006) and molecular aggregates (Gielen et al., 2009) under magnetic fields were reported. In-situ X-ray (Kohama et al., 2007) and neutron (Terada et al., 2008) diffraction measurements under applied magnetic fields were reported. We showed a preliminary result (Matsumoto et al., 2011) of the X-ray diffraction from a MOMS achieved with and without sample rotation in a static magnetic field.

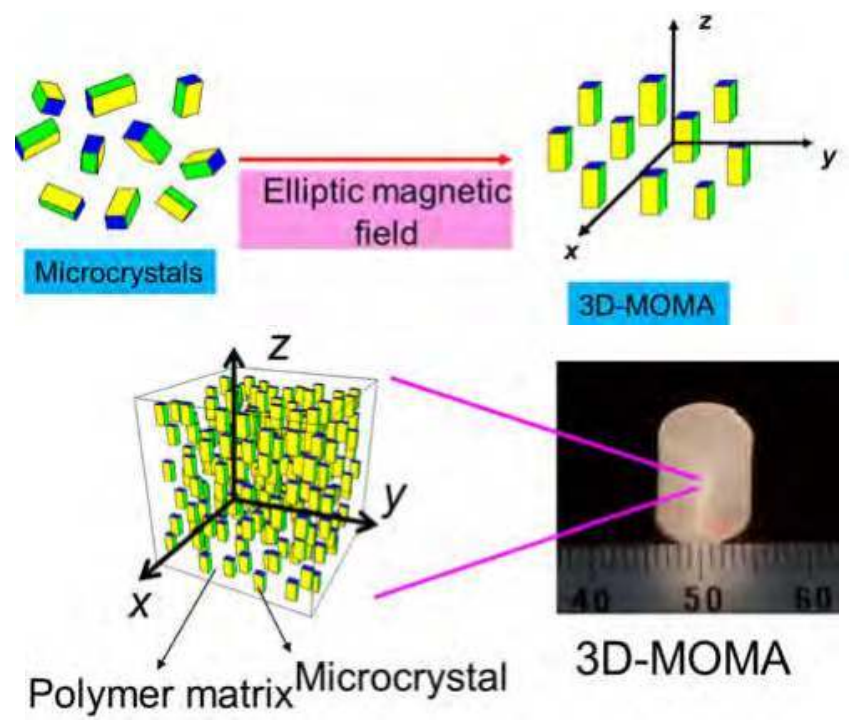

Fig. 1. Schematic of conversion of microcrystals to "three-dimensional magnetically-oriented microcrystal array" (3D-MOMA) or "pseudo single crystal" (PSC). 
We believe that the magnetic method we introduce here is of considerable use for the crystal structure determination of materials that do not grow large sizes necessary for the diffraction measurement. Especially, our method has advantages when applied to single crystal neutron diffraction analyses of proteins where a single crystal sample much larger than that needed for the X-ray diffraction measurement is required.

\section{Theoretical background of preparation of a 3D-MOMA}

A crystal has a diamagnetic susceptibility tensor $\chi$, which is expressed in terms of the principal diamagnetic susceptibility axes $\left(\chi_{3}<\chi_{2}<\chi_{1}<0\right)$. When the crystal is exposed to a magnetic field $\mathbf{B}$, it has a magnetic energy, expressed with respect to the laboratory coordinate system as follows

$$
E=-\left(V / 2 \mu_{0}\right){ }^{\mathbf{t}} \mathbf{B}\left({ }^{\mathrm{t}} \mathbf{A} \chi \mathbf{A}\right) \mathbf{B}
$$

where $\mu_{0}$ is the magnetic permeability of the vacuum, $V$ is the volume of the crystallite, $\mathbf{A}$ is the transformation matrix defined by the Eulerian angles $(\theta, \phi, \varphi)$, and the superscript $t$ indicates the transpose. We consider an applied elliptical magnetic field that periodically changes in direction and intensity on the $x y$ plane as follows:

$$
\mathbf{B}=B \mathrm{t}\left(b_{x} \cos \omega t, b_{y} \sin \omega t, 0\right)
$$

Here, $\omega$ is the angular velocity of the field rotation, $B$ is the intensity of the field, and $b_{x}$ and $b_{y}$ are the longer and shorter axes of the ellipse $\left(b_{y}<b_{x}\right)$, respectively. If $\omega$ is far larger than a certain rate $\tau^{-1}$ (the intrinsic rate of magnetic response of a particle exposed to the static field of the intensity $B$ ) then the easy magnetization axis, $\chi_{1}$, cannot follow the rotation of the field. This condition is called "Rapid Rotation Regime (RRR)", defined as $|\omega \tau|>>1 / 2$, where $\tau$ is expressed as $\tau=6 \mu_{0} \eta / \chi_{\mathrm{a}} B^{2}$ in the case that the particle shape is spherical (T. Kimura et al., 2000) where $\chi_{\mathrm{a}}$ and $\eta$ are magnetic anisotropy and the viscosity of the surrounding medium.

In the RRR, a crystal could be assumed to be placed in an time-averaged magnetic potential expressed by the following equation:

$$
E_{\mathrm{av}}=-(\omega / 2 \pi) \int_{0}^{2 \pi / \omega}\left(V / 2 \mu_{0}\right)^{\mathrm{t}} \mathbf{B}\left({ }^{\mathrm{t}} \mathbf{A} \chi \mathbf{A}\right) \mathbf{B} \mathrm{d} t
$$

Inserting eq (2) into eq (3) and performing the integration, we obtain $E_{\mathrm{av}}$ as an analytical function of the Eulerian angles. Expanding eq (3) around $\theta=\phi=\varphi=0$ and truncating the higher terms, we obtain (T. Kimura et al., 2005)

$$
E_{\mathrm{av}} \approx\left(V / 4 \mu_{0}\right) B^{2} b_{y}{ }^{2}\left(\chi_{2}-\chi_{3}\right) \theta^{2}+\left(V / 4 \mu_{0}\right) B^{2}\left(b_{x}{ }^{2}-b_{y}{ }^{2}\right)\left(\chi_{1}-\chi_{2}\right)(\phi+\varphi)^{2}
$$

where the constant terms are not shown.

Fluctuations of $\theta^{2}$ and $(\phi+\varphi)^{2}$ around the minimum are calculated using the Boltzmann distribution to obtain the expression

$$
<(\Delta \theta)^{2}>\approx 2 \mu_{0} k_{\mathrm{B}} T /\left[B^{2} b_{y}{ }^{2} V\left(\chi_{2}-\chi_{3}\right)\right]
$$




$$
<(\Delta(\phi+\varphi))^{2}>\approx 2 \mu_{0} k_{\mathrm{B}} T /\left[B^{2}\left(b_{x}^{2}-b_{y}{ }^{2}\right) V\left(\chi_{1}-\chi_{2}\right)\right]
$$

where $k_{\mathrm{B}}$ is the Boltzmann constant and $T$ is the temperature. To obtain a good 3D-MOMA, we should minimize and equalize the values of $\left\langle(\Delta \theta)^{2}\right\rangle$ and $\left\langle(\Delta(\phi+\varphi))^{2}\right\rangle$ by appropriately choosing the values of $b_{x}$ and $b_{y}$. The crystallite volume $V$ is also important. The smaller the $V$, the larger the fluctuations. Typically, the size necessary is about the order of micrometers, depending on the field strength used and the magnetic anisotropy of the crystal. To obtain a high quality diffraction pattern, the rotation speed $\omega$ is also important. It should satisfy $|\omega \tau|>>1 / 2$ (RRR) (T. Kimura et al., 2005).

In general, the susceptibility axes of a biaxial crystal do not necessarily coincide with the crystallographic $a, b, c$ axes, except the orthorhombic system (Fig. 2a) (F. Kimura et al., 2010a). The orthorhombic system includes three point groups, that is, 222, $\mathrm{mm} 2$, and $\mathrm{mmm}$. Both for 222 and $\mathrm{mmm}$, three crystallographic axes are 2-fold axes that coincide with the susceptibility axes. As a result, the rotation about the susceptibility axes through an angle of $\pi$ does not create new crystal orientations. On the other hand, $m m 2$ has just one 2 -fold axis. Therefore, the rotation about the susceptibility axes can produce a new orientation.

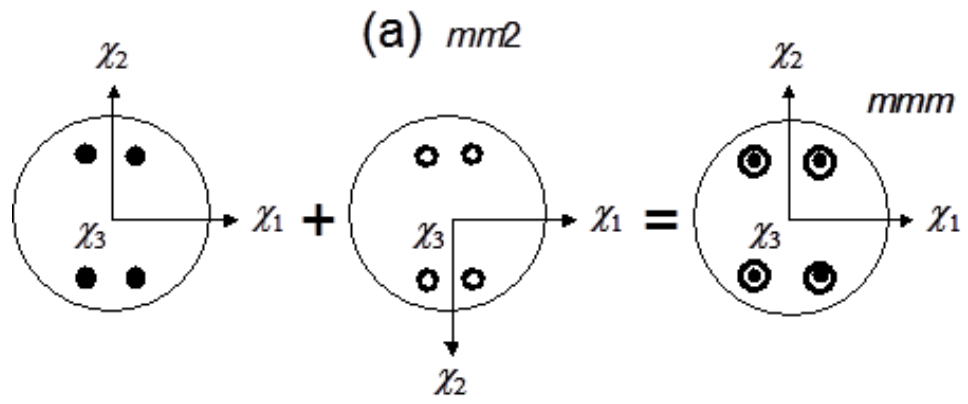

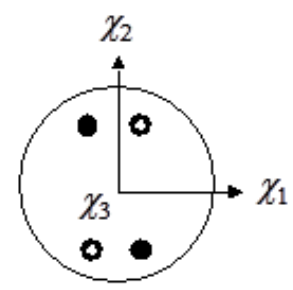

(b) 222

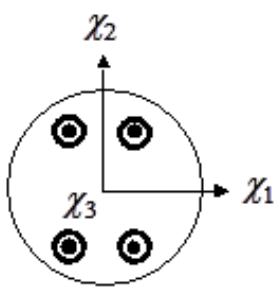

(c) $\mathrm{mmm}$

Fig. 2a. Crystal orientations obtained by a rotation about the susceptibility axis by angle of $\pi$ are magnetically equivalent. The orthorhombic system includes three point groups, $m m 2$, 222 , and $m m m$, as displayed with stereo diagrams. In the case of (a) $m m 2$, an orientation different from the original one is produced by rotation about $\chi_{1}$ or $\chi_{2}$. In a 3D-MOMA, these orientations coexist. For (b) 222 and (c) $\mathrm{mmm}$, the rotations do not alter the original crystal orientation, resulting in a single orientation in a 3D-MOMA. (Reprinted from (F. Kimura et al., 2010a): Cryst. Growth Des. Vol.10(2010) pp. 48-51, No. 1, DOI: 10.1021/cg90132h. Copyright 2010 American Chemical Society) 
For the monoclinic system (point group 2, space group $P 2_{1}$ ), its twofold axis ( $b$ axis) coincides with one of the magnetic susceptibility axes (Nye, 1985). The crystallographic and magnetic axes are shown schematically in Fig. $2 \mathrm{~b}$, where the $b$ axis and $\chi$ axis are placed perpendicular to the plane of the diagram (F. Kimura et al., 2010b). Since the three magnetic susceptibility axes are mutually perpendicular, the other two magnetic susceptibility axes are placed on the plane of the diagram. The $a$ and $c$ axes are on the same plane. However, there is no rule to relate the $\chi$ axes with the $a$ and $c$ axes. If the crystal is rotated by an angle of $\pi$ around each of the magnetic susceptibility axes, four different crystal orientations are obtained, as shown in Figs. $2 b(a)-(d)$. These four orientations have the same magnetic energy when they are placed in a given magnetic field. Because of the twofold symmetry (point group 2) along the $b$ axis, the crystal orientations of Figs. $2 b(a)$ and $2 b(c)$ are identical; those of Figs. $2 b(b)$ and $2 b(d)$ are also identical. Thus, there are two different orientations with the same magnetic energy. In Table 1, possible crystal orientations with an equal magnetic energy are summarized for crystals belonging to the orthorhombic, monoclinic, and triclinic systems.

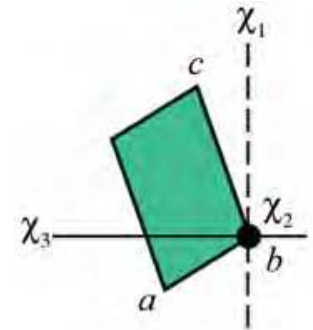

(a)

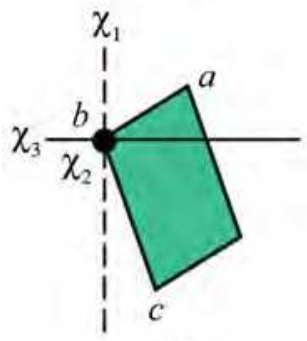

(c)

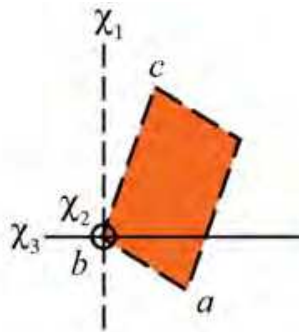

(b)

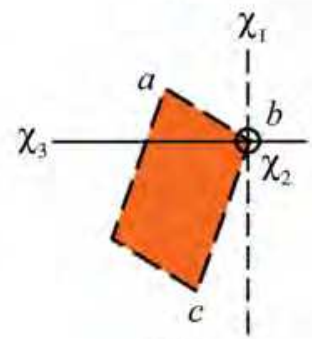

(d)

Fig. 2b. Schematic diagram of a crystal belonging to the monoclinic system (point group 2) showing crystallographic axes and magnetic susceptibility axes. (a) The $b$ axis (twofold axis) and $\chi_{2}$ axis (its direction coincides with that of the $b$ axis) are placed perpendicular to the plane of the diagram. The other crystallographic axes ( $a$ and $c$ axes) and magnetic susceptibility axes ( $\chi_{1}$ and $\chi_{3}$ axes) are placed in the plane. The crystal (a) is rotated by $\pi$ around each of susceptibility axes to produce three additional possible orientations, (b), (c), and (d). All four orientations have the same magnetic energy under a given magnetic field. Because of the symmetry (point group 2), the crystal orientations of (a) and (c) are identical, and those of (b) and (d) are also identical. (Reprinted from (F. Kimura et al., 2010b): J. Appl. Cryst. Vol.143(2010) pp. 151-153, Part 1, DOI: 10.1107/S0021889809048006. Copyright 2010 International Union of Crystallography) 


\begin{tabular}{|l|c|c|c|}
\hline \multirow{2}{*}{ Crystal system } & $\begin{array}{c}\text { Point group of } \\
\text { single crystal }\end{array}$ & $\begin{array}{c}\text { Number of } \\
\text { orientations }\end{array}$ & $\begin{array}{c}\text { Point group of } \\
\text { PSC }\end{array}$ \\
\hline Triclinic & 1 & 4 & 222 \\
\cline { 2 - 4 } & $\overline{1}$ & 4 & $m m m$ \\
\hline \multirow{2}{*}{ Monoclinic } & 2 & 2 & 222 \\
\cline { 2 - 4 } & $m$ & 4 & $m m m$ \\
\cline { 2 - 4 } & $2 / m$ & 2 & $m m m$ \\
\hline \multirow{2}{*}{ Orthorhombic } & 222 & 1 & 222 \\
\cline { 2 - 4 } & $m m 2$ & 2 & $m m m$ \\
\cline { 2 - 4 } & $m m m$ & 1 & $m m m$ \\
\hline
\end{tabular}

Table 1. Number of possible orientations and the resultant point symmetry of Pseudo Single Crystal (PSC) for biaxial crystal systems with different point groups

\section{Magnetic field used to prepare 3D-MOMA}

\subsection{Generation of modulated magnetic field}

A 3D-MOMA of a biaxial crystal was prepared under modulated magnetic fields. In the previous section, the calculation was performed using the elliptical field. In the actual experiment, there are various versions for the elliptical field. We used two different dynamic magnetic fields (T. Kimura et al., 2009b). The first one is an amplitude modulated dynamic field (T. Kimura et al., 2005). This is generated by a four-pole electromagnet, in which one pair of poles generates a $B_{x}$ field strength sinusoidally oscillating at $\omega$, and the other generates $B_{y}$ field strength oscillating at the same frequency as the previous one but with a phase shift of $\pi / 2$. This combination created a magnetic field expressed as $\left(B_{x} \cos \omega t, B_{y} \sin \omega t, 0\right)$, shown in Fig. 3. The second one is a frequency modulated dynamic magnetic field (T. Kimura et al., 2006). In this setup, a sample is non-uniformly rotated in a uniform static magnetic field. A sample-rotating unit was placed in a uniform horizontal magnetic field, shown in Fig. 4. The rotation axis was vertical. The rotation was not uniform. In the figure, the $x-, y-$, and $z$-axes are laboratory coordinates and the $x^{\prime}-, y^{\prime}-$, and $z^{\prime}$-axes are imbedded in the rotating plate. The $x$-axis is parallel to the magnetic field. The $z^{\prime}$ - and $z$-axes are parallel to vertical direction. The angle between the $x^{\prime}$-axis before rotating and $x$-axis is $\alpha / 2$. The $x^{\prime}$-axis was rotating at the angular velocity $\omega_{\mathrm{s}}$ between $360-\alpha / 2$ and $\alpha / 2$ degree, and between $180-\alpha / 2$ and $180+\alpha / 2$ degree. On the other hand, it was rotating at the angular verocity $\omega_{\mathrm{q}}\left(>\omega_{\mathrm{s}}\right)$ between $\alpha / 2$ and $180-\alpha / 2$ degree, and between $180+\alpha / 2$ and $360-\alpha / 2$ degree.

The three parameters, $\omega_{s}, \omega_{\mathrm{q}}$, and $\alpha$, must be selected appropriately in order to obtain sharp diffraction spots. The half-width of a spot is a result of the fluctuations of the $\chi_{1}$ and $\chi_{3}$ axes about the $x^{\prime}$ and $z^{\prime}$ axes, respectively. In terms of diffraction analysis, it is advantageous that the magnitudes of the two fluctuations are equal. 

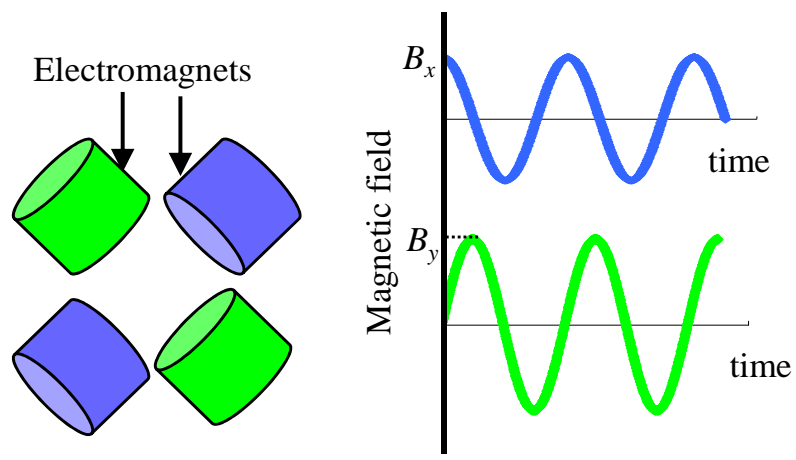

Fig. 3. Generation of amplitude modulated magnetic field. Two pairs of magnets generate $B_{x} \cos \omega t$ and $B_{y} \sin \omega t$ resulting in an elliptical magnetic field.

\section{Superconducting magnet}

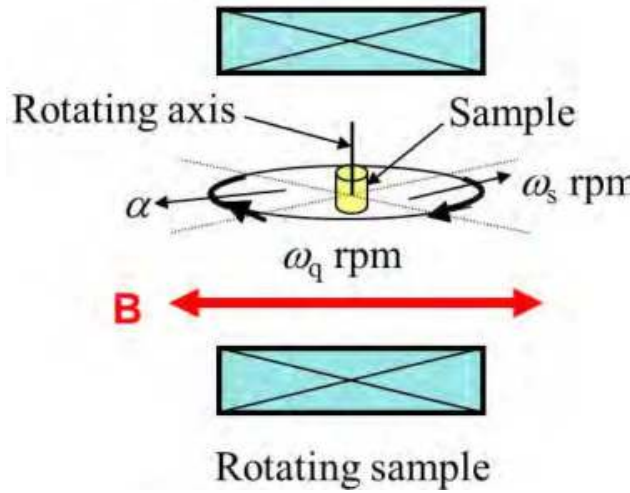

(a)

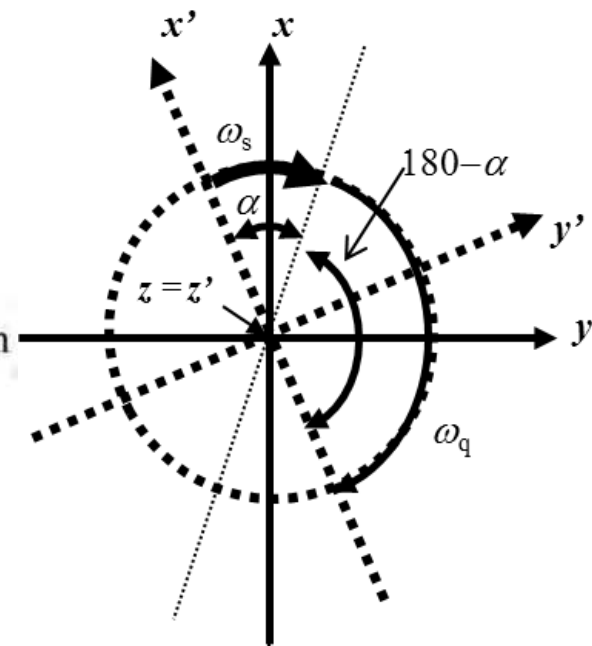

(b)

Fig. 4. (a) Generation of modulated magnetic field by rotating a sample in a static field. Rotation speed is switched. (b) Diagram illustrating the frequency-modulated sample rotation. The $x-, y$-, and $z$-axes are laboratory coordinates; the $x$ - and $y$-axes are on the horizontal plane and the $z$ axis is on the vertical plane. The $x^{\prime}-, y^{\prime}-$, and $z^{\prime}$-axes are imbedded in the sample that is rotated around the $z^{\prime}$-axis (the $z$-axis coincides with the $z^{\prime}$-axis). The rotation is performed non-uniformly so that the condition $\omega_{\mathrm{s}}<\omega_{\mathrm{q}}$ is satisfied. The $x$-axis coincides with the direction of the magnetic field.

\section{Sample preparation}

\subsection{Preparation of 3D - MOMA}

Micro-crystallites were mixed with a UV (Ultra Violet) curable monomer. The concentration of the crystallites in the monomer is $10-30 \mathrm{v} / \mathrm{v} \%$. The suspension is poured into any size of 
plastic container, exposed to a dynamic magnetic field, followed by UV light irradiation to polymerize the resin precursor to fix the alignment. See experimental details in Table 2.

\subsection{Preparation of MOMS}

Typical experimental setting (KU model $\chi 10-1$ ) is shown in Fig. 5. Sample suspension is poured into a capillary, and then it is placed on a rotating unit equipped with a pair of magnets.

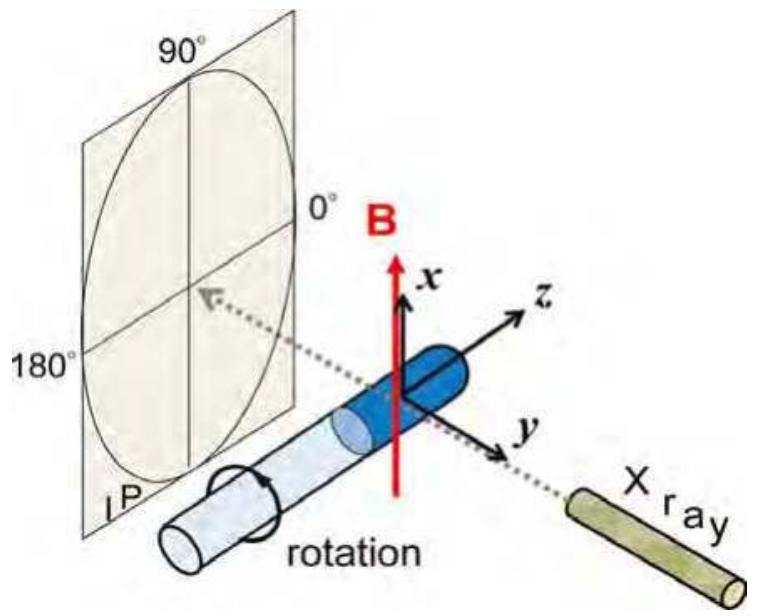

Fig. 5. Experimental setting of in-situ X-ray diffraction measurement of a microcrystal suspension. A glass capillary containing the suspension is rotated at the rotation speed $\omega$ about the $z$-axis. The magnetic field $\mathbf{B}$ is applied parallel to the $x$-axis. The $\mathrm{X}$-ray beam is impinged from the $y$ direction, and the diffractions are detected by an imaging plate (IP). The azimuthal $\beta$ angle is indicated. (Reprinted from (Matsumoto et al., 2011): Cryst. Growth Des. Vol.11(2011) pp. 945-948, No. 4, DOI: 10.1021/cg200090u. Copyright 2011 American Chemical Society)

\section{X-ray results}

We fabricated 3D-MOMAs using frequency modulated magnetic field except $\mathrm{LiCoPO}_{4}$. We fabricated 3D-MOMAs of $\mathrm{LiCoPO}_{4}$ using both amplitude and frequency modulated magnetic fields. Alignment of these 3D-MOMAs are almost the same. Here, we only show the results obtained from the MOMA using frequency modulated magnetic field.

\subsection{Alanine (T. Kimura et al., 2006)}

The top figure in Fig. 6 shows the XRD powder pattern obtained from the experiment in which magnetic field was not applied. A sparse ring pattern is observed in this case. Figures 6(a), (b) and (c) show the XRD patterns of the obtained pseudo single crystal. Patterns in (a), (b), and (c) verify the alignment of the $a, b$, and $c$ axes, respectively. The $y^{\prime}, z^{\prime}$, and $x^{\prime}$ axes imbedded on the sample correspond to the $a, b$, and $c$ axes, respectively. This indicates that the $b$ and $c$ axes correspond to the hard and easy magnetization axes, respectively. 


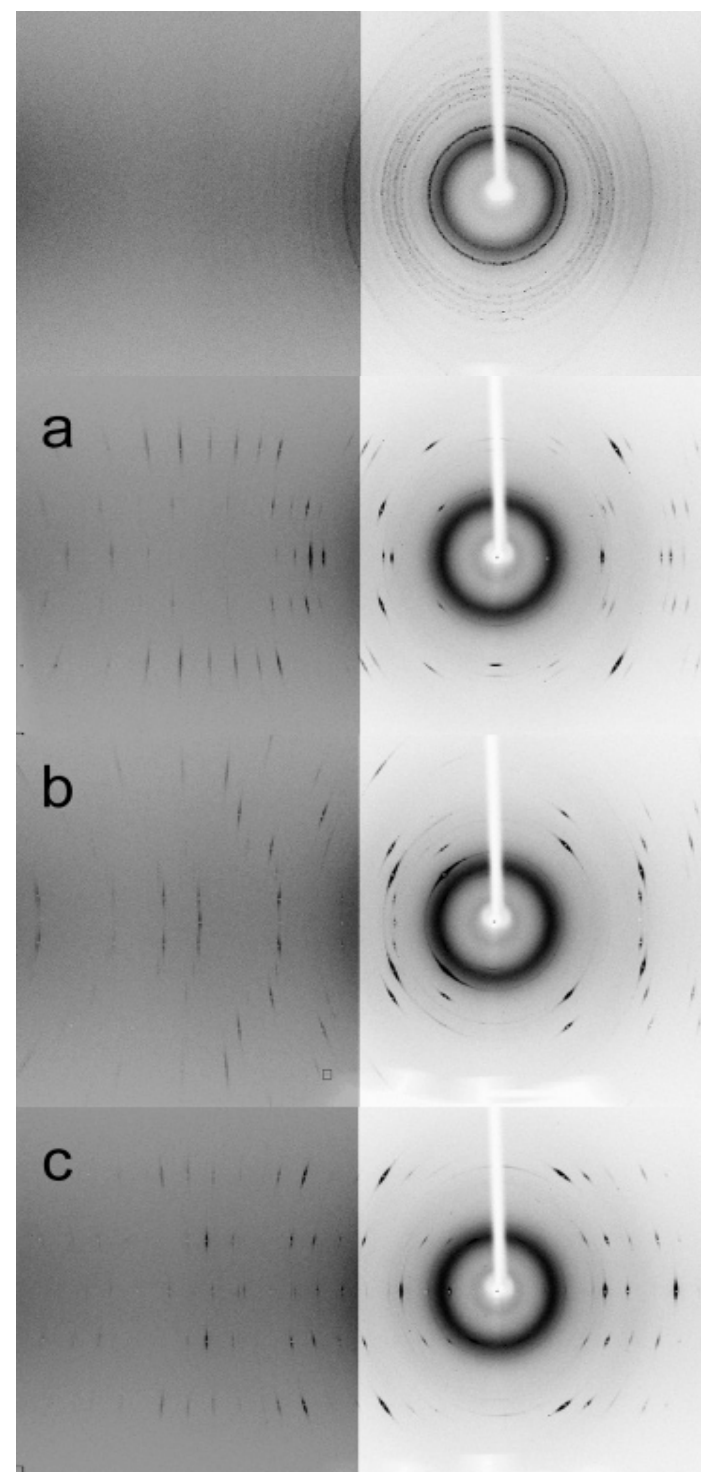

Fig. 6. X-ray diffraction pattern obtained for the sample prepared without the application of a magnetic field(top). X-ray diffraction patterns obtained for the L-alanine pseudo-single crystal sample. Patterns in (a), (b), and (c) were obtained with the alignment of the $a, b$, and $c$ axes, respectively, perpendicular to the X-ray beam; this is achieved by using an automatic crystal axis alignment system. An oscillation of $10^{\circ}$ was applied around the aligned axis. Some of the assignments were performed using a software program. Contrasts are different between left and right halves. (Reprinted from (T. Kimura et al., 2006): Langmuir Vol.22(2006) pp. 3464-3466. No. 8, DOI: 10.1021/la053479m. Copyright 2006 American Chemical Society) 
The diffraction spots are broad compared to those from a real single crystal, but they are sufficiently distinguishable to be assigned by the software program. Since the lattice parameter is the largest for the $b$ axis, the spots in Fig. 6(b) are relatively less resolved. The half widths of the spots obtained by using an azimuthal plot are ca. $3^{\circ}$ for most of the spots in all of the patterns in Figs. 6 (a), (b), and (c). Incidentally, the half widths for an actual original single crystal are ca. $0.5^{\circ}$. The fluctuations in $\theta$ and $\varphi+\psi$ are complex functions in the case of the frequency-modulated magnetic field used in the present study. However, they can be roughly estimated by using eqs (5) and (6). Using the values of $B=5 \mathrm{~T}, b_{x}=1$, $b_{y}=0.5, T=300 \mathrm{~K}$, and a rough estimation of $\chi_{1-} \chi_{2}$ and $\chi_{2}-\chi_{3}=10^{-7}$, we can obtain the estimation for the fluctuation of $\theta$ and $\phi+\varphi$ ca. $1^{\circ}$ for $V=1000 \mu \mathrm{m}^{3}$. This value should be compared with the half widths of the diffraction spots. The experimental values are larger than those obtained by the theoretical estimation. Using the values of the viscosity $(\eta=1.2 \mathrm{~Pa}$ s) and the shape factor ( $F=0.064$, corresponding to the assumed aspect ratio of 11 for crystallites), we can obtain the estimation, $\tau=1 \mathrm{~min}$. The time required for the completion of the alignment might be estimated as five times of $\tau$, i.e., ca. $5 \mathrm{~min}$. This value is far shorter than $2 \mathrm{~h}$ actually applied in the experiment. These discrepancies have several possible explanations, including the existence of smaller crystallites, imperfect dispersion, shrinkage of the resin during the UV cure, the flow caused by sample rotation, insufficient rotation speed, imperfect switching from one rotation to the other, etc.

\section{2 $\mathrm{LiCoPO}_{4}$ (T. Kimura et al., 2009b)}

A typical diffraction of the 3D-MOMA image is shown in Fig. 7. The estimated mosaicity is $3.9^{\circ}$, which is larger than that observed for normal single crystals on the same instrument (around $0.8^{\circ}$ ). The cell constants and an orientation matrix for data collection corresponded to a primitive orthorhombic cell with dimensions $a=10.202$ (6), $b=5.918$ (3), $c=4.709$ (2) $\AA$, $V=284.3(3) \AA^{3}$ and $Z=4$. The space group was determined to be Pnma (No. 62). These results are the same as those reported by Kubel (1994), who used a $0.090 \times 0.108 \times 0.158 \mathrm{~mm}$ single crystal. The structure was solved by direct methods and expanded using Fourier techniques. The present result was compared with that from the literature, showing that the atomic coordinates determined in this study are in excellent agreement with those determined using a traditional single crystal. The $R 1$ and $w R 2$ values were 6.59 and $16.8 \%$, respectively.

\subsection{Sucrose (F. Kimura et al., 2010b)}

The sucrose crystal belongs to the monoclinic system (point group 2, space group $P 2_{1}$ ). Assuming an initial random orientation of crystallites in a suspension, the probability of finding two different orientations with the same magnetic energy is equal. These two orientations produce a diffraction pattern similar to that produced by a twin crystal. The diffraction image was analyzed using software designed for twin structures. The cell constants correspond to a primitive monoclinic cell with dimensions $a=7.7735(12), b=$ 8.7169(13), $c=10.8765(17) \AA, \beta=102.936(4)^{\circ}, V=718.29(19) \AA^{3}$ and $Z=2$. The space group was determined to be $P 2_{1}$ (No. 4). The structure was solved by direct methods and expanded using Fourier techniques. The $R 1$ and $w R 2$ values were 7.88 and $17.25 \%$, respectively. 

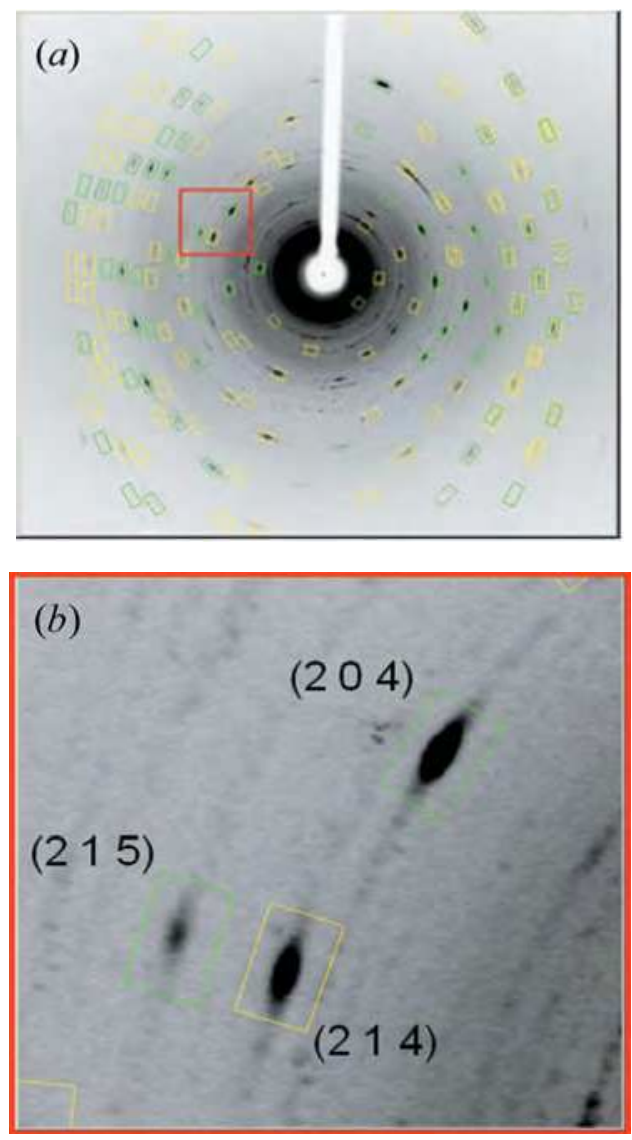

Fig. 7. (a) A typical X-ray diffraction image (oscillation angle $5^{\circ}$ ) of a pseudo single crystal of $\mathrm{LiCoPO}_{4}$ is shown with diffraction spots enclosed by prediction rectangles. (b) An enlarged view of some diffraction spots is shown with Miller indices. (Reprinted from (T. Kimura et al., 2009b): J. Appl. Cryst. Vol.142(2009) pp. 535-537, Part 3, DOI:

10.1107/S0021889809013430. Copyright 2009 International Union of Crystallography)

\begin{tabular}{|l|c|c|c|c|}
\hline & applied magnetic field / T & $\alpha /$ degree & $\omega_{\mathrm{s}} / \mathrm{rpm}$ & $\omega_{\mathrm{q}} / \mathrm{rpm}$ \\
\hline L-alanine for X-ray & 5 & 90 & 5 & 25 \\
L-alanine for neutron & 8 & 90 & 40 & 132 \\
\hline LiCoPO $_{4}$ & 0.3 & 90 & 10 & 60 \\
\hline Sucrose & 12 & 90 & 10 & 60 \\
\hline Lysozyme & 8 & 10 & 10 & 60 \\
& & 10 & 30 & 100 \\
& & 20 & 10 & 60 \\
\hline
\end{tabular}

Table 2. Preparation condition of MOMAs 
In Fig. 8, the structure solved from the MOMA is compared with that reported previously (Hanson et al., 1973). They show a good agreement. The twin structure is shown in Fig. 9. The magnetic susceptibility axes, as discussed previously (Fig. 9), are also shown. In principle, these magnetic susceptibility axes are attributed to each of the easy (largest) $\chi_{1}$, hard (smallest) $\chi_{3}$, and intermediate (medium) $\chi_{2}$ magnetic susceptibility axes. The angle between the magnetic axis, $\chi_{1}$, and crystallographic $c$ axis is determined to be ca $16^{\circ}$. This value is different from a previously reported value of $-1^{\circ} 5^{\prime}$ (Finke, 1909). The reason for the difference is unclear at present.

\subsection{Lyzozyme (F. Kimura et al., 2011)}

The fluctuations of the $\chi_{1}$ and $\chi_{3}$ axes were estimated from the half-widths determined from the rocking curve and the azimuthal $\beta$ scan, respectively. The rotation axis of the $\omega$ scan coincided with the $\chi_{3}$ axis. The data was collected using $\omega$ scans at $1.0^{\circ}$ steps. The three parameters, $\omega_{s}, \omega_{\mathrm{q}}$, and $\alpha$, must be selected appropriately in order to obtain sharp diffraction spots. The half-width of a spot is a result of the fluctuations of the $\chi_{1}$ and $\chi_{3}$ axes about the $x^{\prime}$ and $z^{\prime}$ - axes, respectively. In terms of diffraction analysis, it is advantageous that the magnitudes of the two fluctuations are equal. Sample rotation speed $\omega_{s}>1 /(2 \tau)$ should be met (Rapid Rotation Regime (RRR)). Next, an appropriate choice of $\alpha$ and $\omega_{\mathrm{q}}$ is necessary in order to minimize and equalize the fluctuations of the $\chi_{1}$ and $\chi_{3}$ axes.

In alanine, $\mathrm{LiCoPO}_{4}$, and sucrose studies describing the single-crystal analysis of MOMAs, $\alpha=90^{\circ}$ was appropriate. However, a theoretical study (T. Kimura, 2009a) shows that the equalization cannot be achieved for some sets of $\left(\chi_{1}, \chi_{2}, \chi_{3}\right)$ if $\alpha$ is fixed to $90^{\circ}$. Since the three values of the magnetic susceptibility of lysozyme are unknown, we need to find out an appropriate value of $\alpha$ by trial and error. Table 3 summarizes the half-widths of a diffraction spot ((400) diffraction) obtained from MOMAs fabricated with some sets of parameters $\left(\omega_{\mathrm{s}}, \omega_{\mathrm{q}}, \alpha\right)$.

\begin{tabular}{|c|c|c|c|c|}
\hline$\alpha /$ degree & $\omega_{\mathrm{s}} / \mathrm{rpm}$ & $\omega_{\mathrm{q}} / \mathrm{rpm}$ & Fluctuation of $\chi_{1} /$ degree & Fluctuation of $\chi_{3} /$ degree \\
\hline 10 & 10 & 60 & 4.5 & 4.3 \\
\hline 10 & 30 & 100 & 5.7 & NA \\
\hline 20 & 10 & 60 & 6 & 5.1 \\
\hline
\end{tabular}

Table 3. Fluctuation of $\chi_{1}$ and $\chi_{3}$ for three sets of parameters $\left(\omega_{\mathrm{s}}, \omega_{\mathrm{q}}, \alpha\right)$, determined using the (400) diffraction spot.

We tested several combinations of the parameters, and $\alpha=10^{\circ}, \omega_{\mathrm{s}}=10 \mathrm{rpm}$, and $\omega_{\mathrm{q}}=60 \mathrm{rpm}$ was found to be the most suitable regarding minimization and equalization of the halfwidths. This condition was therefore chosen for the preparation of a MOMA for X-ray analysis. In Fig. 10, photograph and microphotograph of fabricated lysozyme MOMA are shown. In Fig. 11, diffraction patterns taken from three directions are shown. Well-separated diffraction spots were obtained. The directions of the magnetic axes as deduced from the preparation procedure are indicated in the figure. From the results of the indexing described in the next paragraph, the crystal belongs to the orthorhombic system. For this crystal 


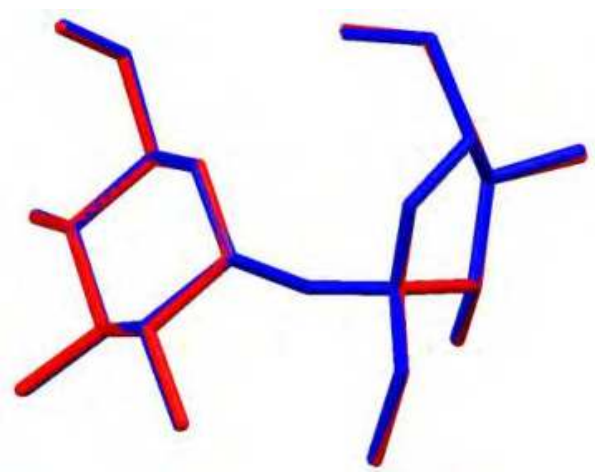

Fig. 8. Comparison of the structure determined in this study (blue) and the structure reported previously (red). (Reprinted from (F. Kimura et al., 2010b): J. Appl. Cryst. Vol.143(2010) pp. 151-153, Part 1, DOI: 10.1107/S0021889809048006. Copyright 2010 International Union of Crystallography)

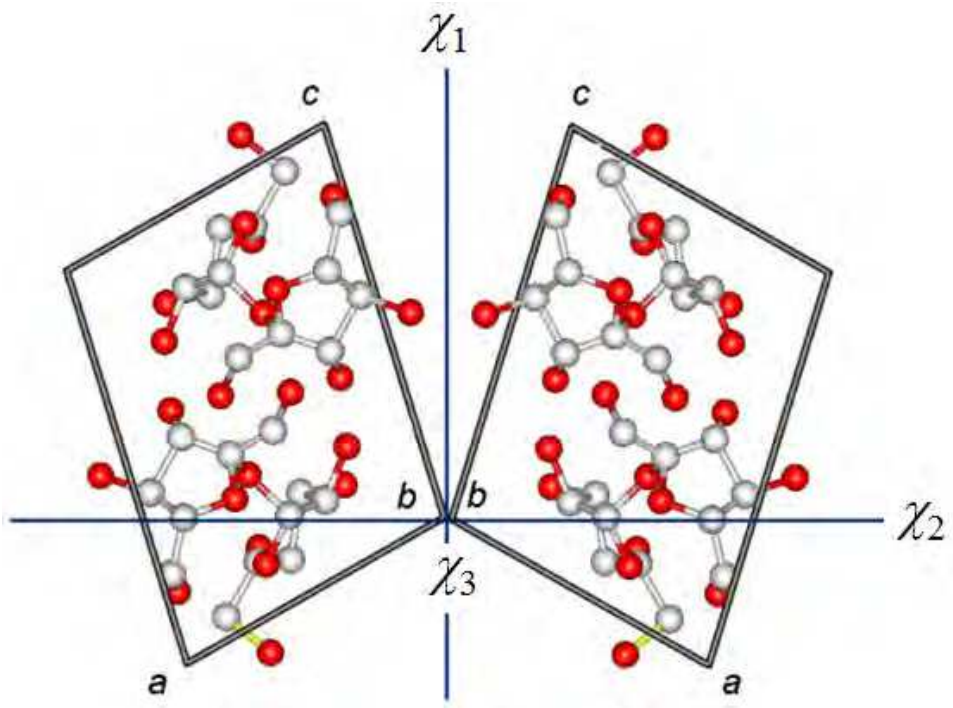

Fig. 9. Twin structure of a 3D-MOMA of sucrose. The $b$ axis and one of the susceptibility axes (here denoted as $\left.\chi_{3}\right)$ are placed perpendicular to the plane of the diagram. The other axes $\left(\chi_{1}\right.$ and $\left.\chi_{2}\right)$ are placed in the plane. The angle between the $\mathrm{c}$ axis and the $\chi_{1}$ axis is ca $16^{\circ}$. (Reprinted from (F. Kimura et al., 2010b): J. Appl. Cryst. Vol.143(2010) pp. 151-153, Part 1, DOI: 10.1107/S0021889809048006. Copyright 2010 International Union of Crystallography)

system, the magnetic axes correspond to the crystallographic axes. From the figure, we find that the cell dimensions increase in the order $\chi_{1}, \chi_{2}$, and $\chi_{3}$. Combining the indexing results, we conclude that $\chi_{1}=c, \chi_{2}=a$, and $\chi_{3}=b$. The alignment of the $c$ axis parallel to the magnetic field has been reported for orthorhombic lysozyme crystals.( Sato et al., 2000). Figure 12 shows a detailed diffraction pattern with resolution rings. 

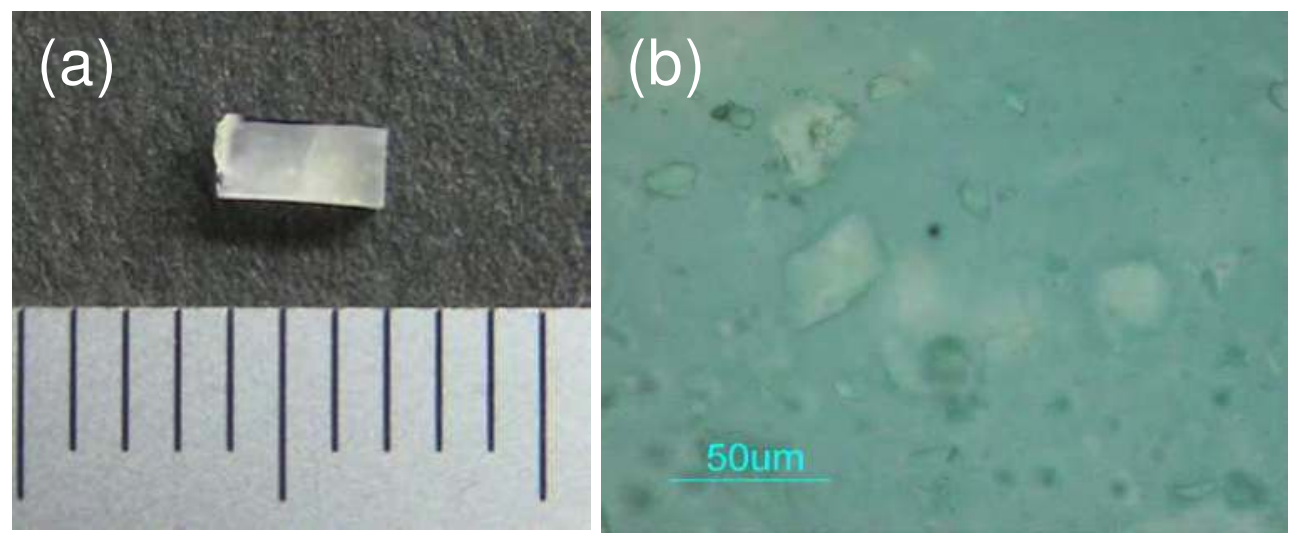

Fig. 10. (a) Photograph (a division: $1 \mathrm{~mm}$ ) of a magnetically oriented microcrystal array (MOMA) of lysozyme. (b) Microphotograph of MOMA that is composed of various sizes of microcrystals. (Reprinted from (F. Kimura et al., 2011): Cryst. Growth Des. Vol.11(2011) pp. 12-15, No. 1, DOI: 10.1021/cg100790r. Copyright 2010 American Chemical Society)
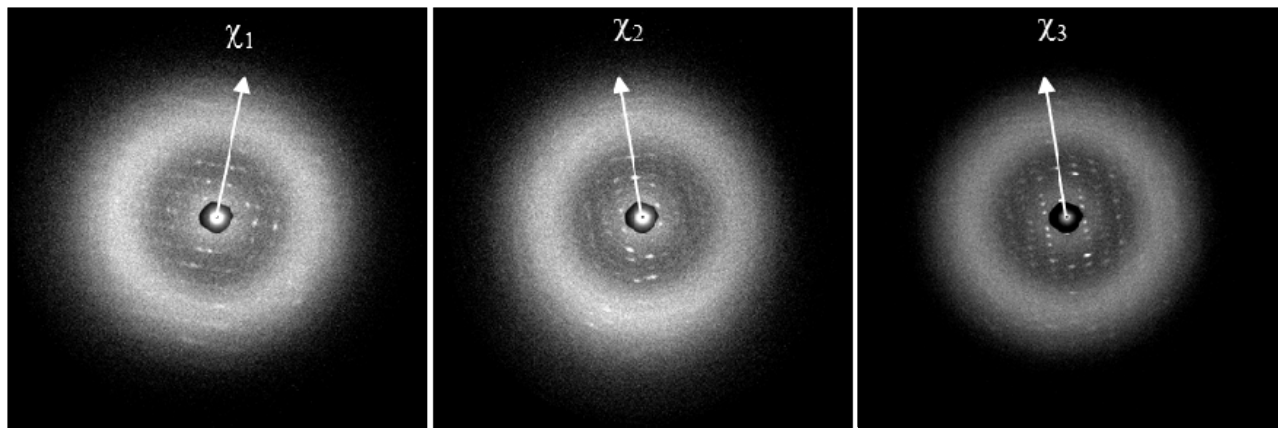

Fig. 11. X-ray diffraction of a lysozyme MOMA taken from three different orthogonal directions. (Reprinted from (F. Kimura et al., 2011): Cryst. Growth Des. Vol.11(2011) pp. 1215, No. 1, DOI: 10.1021/cg100790r. Copyright 2010 American Chemical Society)

The X-ray results are summarized in Table 4 . The indexing was determined as follows: space group, $P 2{ }_{1} 22_{1}{ }_{1}$; lattice constants, $a=51.26 \AA, b=59.79 \AA, c=29.95 \AA$, and $V=91803 \AA^{3}$. This data was compared with that reported on the lysozyme single crystal (PDB code 2ZQ4) in Table 5. The cell dimensions obtained in this study are shorter than those reported in the literature. The shrinkage was attributed to the dehydration of the crystal. X-ray diffraction of dehydrated lysozyme crystals in triclinic (Kachalova et al., 1991) and monoclinic (Nagendra et al., 1998) forms has been reported. The graphical display is shown in Fig. 13 for easy comparison of the MOMA with the reported structure (PDB code 2ZQ4). A comparison of the CR positions between the present result and 2ZQ4 gave rmsd=0.755 $\AA$, indicating that the shrinkage of the lattice was mainly due to the loss of water molecules and that the protein chain conformation remained essentially unchanged. 


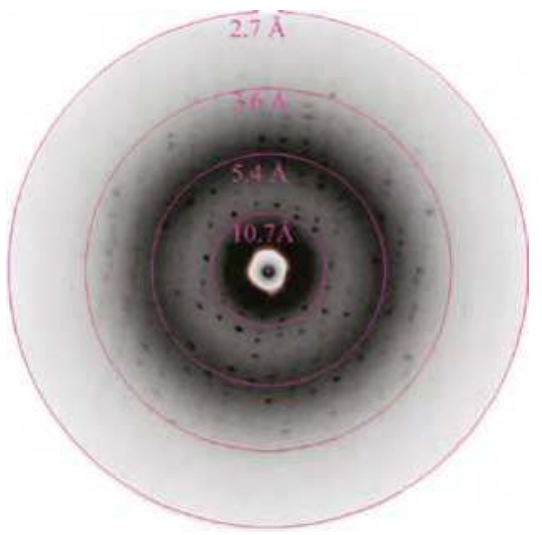

Fig. 12. X-ray diffraction image of the lysozyme MOMAwith resolution rings indicated. (Reprinted from (F. Kimura et al., 2011): Cryst. Growth Des. Vol.11(2011) pp. 12-15, No. 1, DOI: 10.1021/cg100790r. Copyright 2010 American Chemical Society)

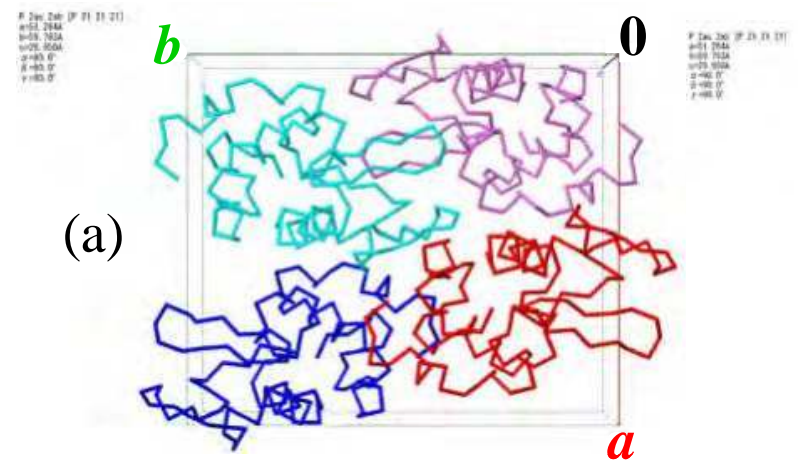

(b)
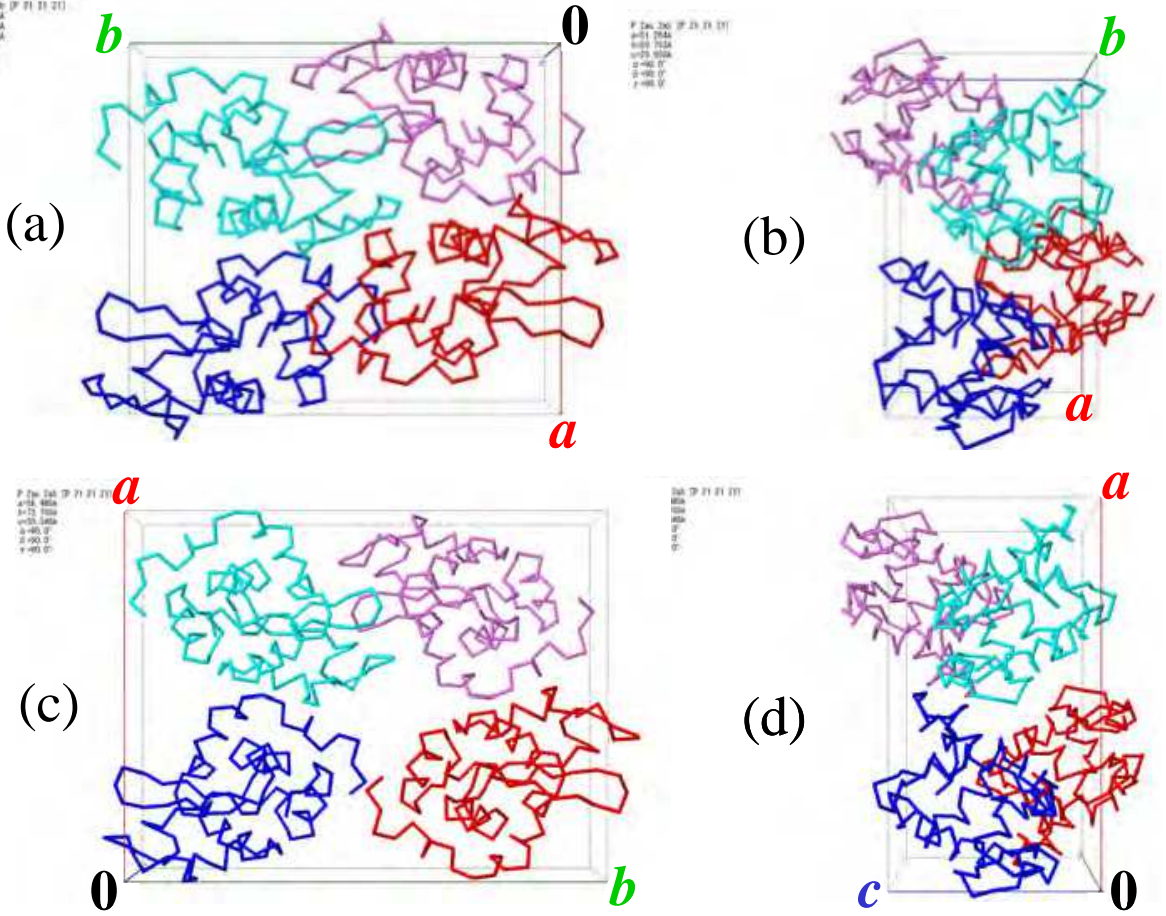

Fig. 13. Comparison of the crystal structures of lysozyme: ( $a$ and $b$ ) the structure determined through a MOMA prepared in the present study; (c and d) the structure reproduced from the database (PDB code 2ZQ4). (Reprinted from (F. Kimura et al., 2011): Cryst. Growth Des. Vol.11(2011) pp. 12-15, No. 1, DOI: 10.1021/cg100790r. Copyright 2010 American Chemical Society) 
A. Crystal data

Wavelength $(\AA)$

Space group

Cell dimensions

$$
\begin{aligned}
& \text { a }(\AA) \\
& \text { b }(\AA) \\
& \text { c }(\AA) \\
& \text { V }\left(\AA^{3}\right)
\end{aligned}
$$

Observed reflections

Resolution $(\AA)$

Independent reflections

completeness (\%)

$\mathrm{R}_{\text {sym }}(\%)$

Redundancy

Mean I/ $\sigma$

B. Refinement statistics

Resolution limits $(\AA)$

No. of reflections used

completeness (\%)

No. of protein atoms

No. of solvent molecules

Final R-factor

Free $\mathrm{R}$ value

Average B-factor $\left(\AA^{2}\right)$

r.m.s. deviation from ideal geometry

$$
\begin{aligned}
& \text { Bond distances }(\AA) \\
& \text { Bond angles (deg.) } \\
& \text { Dihedrals (deg.) }
\end{aligned}
$$

\subsection{8}

$\mathrm{P} 2{ }_{1} 2_{1} 2_{1}$

51.26

59.79

29.95

91,803

10,604

$26.77-3.00(3.16-3.00)$

$1,828(250)$

89.6 (88.6)

$19.4(48.7)$

$5.8(5.5)$

$9.3(2.7)$

25.86 - $3.00(3.78-3.00)$

$2,989(1,423)$

$84.4(81)$

1,001

0

$0.215(0.253)$

$0.270(0.292)$

51.0

0.004

0.743

22.596

Table 4. Summary of data collection and refinement for lysozyme MOMA. The values in highest resolution bin were indicated in the parentheses. 


\section{Neutron diffraction data}

\subsection{Alanine (F. Kimura et al., 2010a)}

In measuring the pole figure, a 3D-MOMA sample was set on a goniometer approximately in the direction shown in Fig. 14(a). The angles $\chi$ and $\varphi$ correspond to the operation angles of the goniometer. The angle $\varphi$ runs from 0 to $180^{\circ}$ at a given value of the angle $\chi$ that runs from 0 to $-180^{\circ}$. At this setting, the diffractions corresponding to the (040), (002), (120), etc. are expected to appear at locations shown in the figure. In Fig. 14(b), the measured pole figure is displayed. Here, all the diffraction spots are displayed in the same figure. A half sphere was scanned so that spots for $\{120\}$ were all observed. These spots appear around $\chi=0,-180^{\circ}$ and $\varphi=45,135^{\circ}$ because $a / b=1 / 2$. On the other hand, a spot for (040) was only scanned on one side. Instead, a close examination of (040) was made by scanning in the vicinity of $\chi=0^{\circ}$ and $\varphi=90^{\circ}$. A contour plot of the intensity of (040) is shown in Fig. 15 as a function of $\chi$ and $\varphi$. The average fwhm over two angles is ca. $4^{\circ}$. A $2 \theta$ profile for the (040) diffraction is shown in Fig. 16 as a typical example. The peak is clearly distinguished from the background. This high signal-to-noise ratio was unexpected because no care was taken to reduce the background incoherent scattering when choosing the resin precursor. This might be attributed to the fact that the coherent diffraction is extremely increased because the microcrystals are aligned three-dimensionally.

(a)

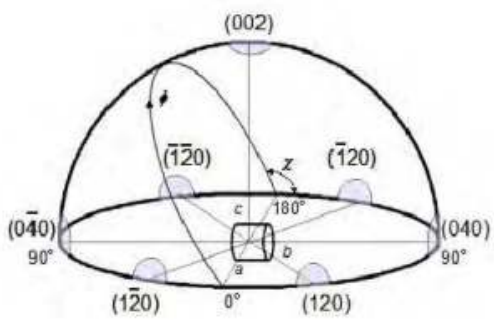

(b)

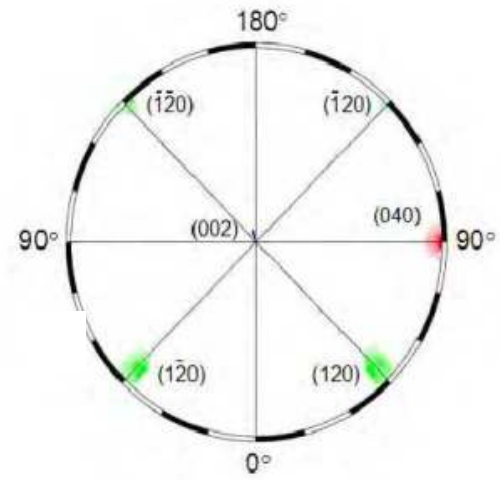

Fig. 14. (a) Sample setting of the L-alanine PSC and the expected diffraction spots. Angle $\chi$ runs from 0 to $-180^{\circ}$ and $\varphi$ runs from 0 to $180^{\circ}$. (b) Pole figure obtained for the PSC to be compared with (a). (Reprinted from (F. Kimura et al., 2010a): Cryst. Growth Des.Vol.10(2010) pp. 48-51. No. 1, DOI: 10.1021/cg90132h. Copyright 2010 American Chemical Society) 


\begin{tabular}{|c|c|c|}
\hline Sample & MOMA & Single crystal \\
\hline PDB Code & present work & $P 2{ }_{1} 2_{1} 2_{1}$ \\
\hline Space group & $P 2{ }_{1} 2_{1} 2_{1}$ & \\
\hline Cell dimensions & & 56.48 \\
\hline$a(\AA)$ & 51.26 & 73.76 \\
\hline$b(\AA)$ & 59.79 & 30.54 \\
\hline$c(\AA)$ & 29.95 & 127,229 \\
\hline$V\left(\AA \AA^{3}\right)$ & 91,803 & \\
\hline
\end{tabular}

Table 5. Comparison of crystal data of lysozyme.

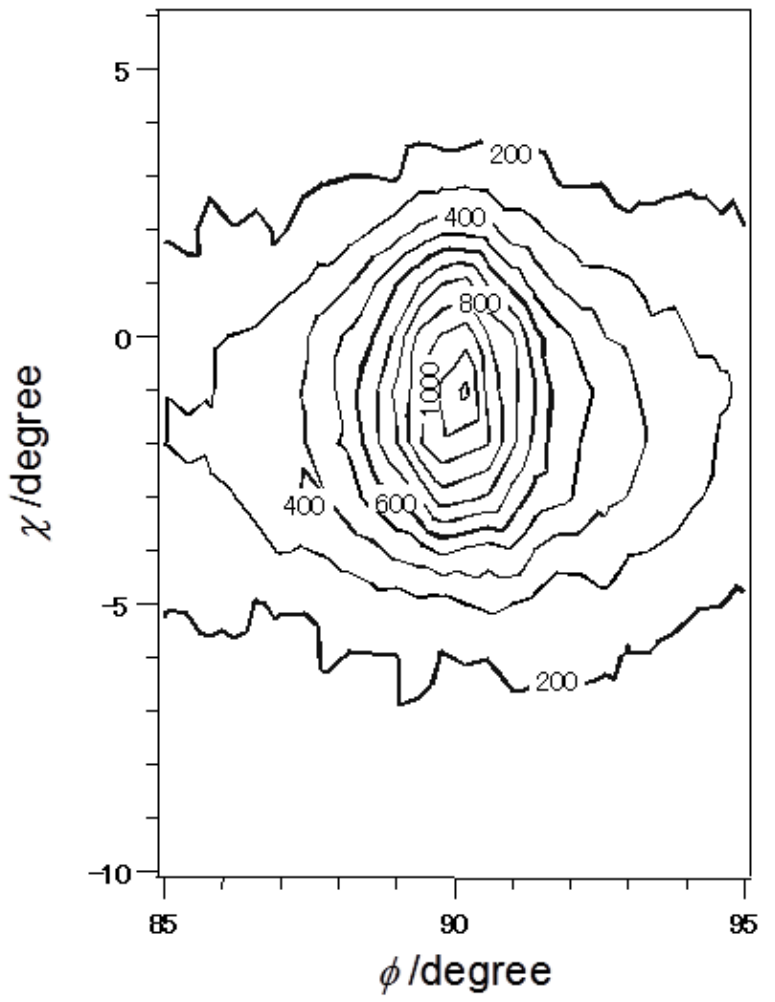

Fig. 15. Contour plot of the diffraction spot for (040) shown in Fig. 4(b) as a function of $\chi$ and $\phi$. Half maximum of the intensity is ca. 600 from which the average fwhm is estimated to be ca. $4^{\circ}$. (Reprinted from (F. Kimura et al., 2010a): Cryst. Growth Des. Vol.10(2010) pp. 48-51, No. 1, DOI: 10.1021/cg90132h. Copyright 2010 American Chemical Society) 


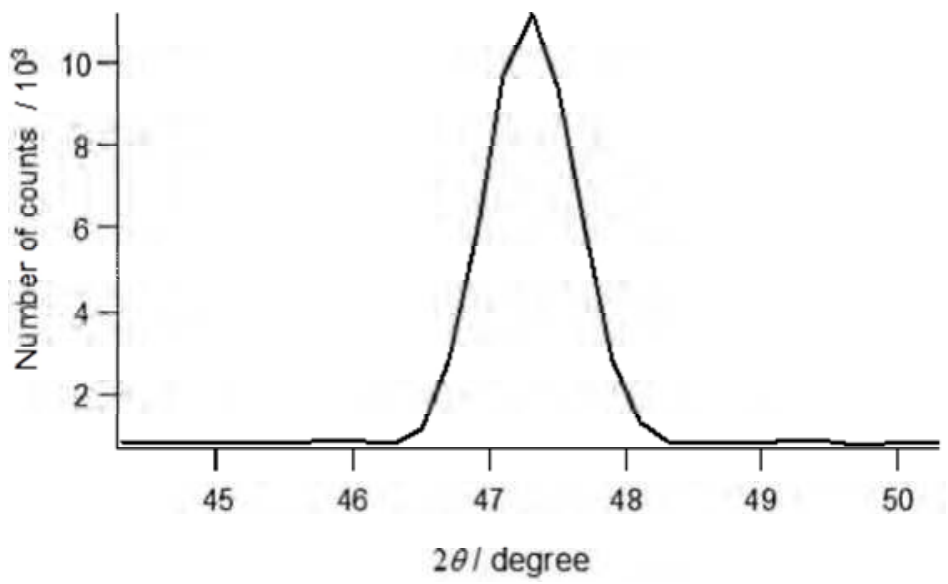

Fig. 16. Diffraction profile for the (040) plane. Baseline is not subtracted. (Reprinted from (F. Kimura et al., 2010a): Cryst. Growth Des. Vol.10(2010) pp. 48-51, No. 1, DOI: 10.1021/cg90132h. Copyright 2010 American Chemical Society)

After subtraction of the baseline, the intensity of the peak is calculated by integration and corrected for Lorentz factor; no absorption correction was performed. The structure refinement was performed with Shelxl-97 using the crystallographic data of neutron diffraction of L-alanine reported in the literature (Wilson et al., 2005), where the atom coordinates, the temperature factors, and the anisotropic temperature factors were fixed. The results are summarized in Table 6 . The values of $R 1$ and $w R 2$ are 0.184 and 0.368 , respectively. In the present preliminary study, the integration was performed only along the $2 \theta$ direction, and the integration with respect to $\chi$ and $\varphi$ directions was not performed. In addition, absorption correction was not made. Hence, the intensity data could include some error. However, the experimental and calculated results appear to agree satisfactorily, indicating that the MOMA can provide diffraction data that will lead to a successful structure analysis.

\section{Possibility of MOMS for application of structure analysis (Matsumoto et al., 2011)}

Figure 17 shows the diffraction patterns obtained for $\omega=0 \mathrm{rpm}$ (static) and $20 \mathrm{rpm}$. At $\omega=0$ rpm, the $\{002\}$ diffractions did not appear, and the $\{120\}$ diffractions appeared on the equator. This suggests that the reciprocal vectors $\mathrm{G}\{002\}$ are aligned in the direction of the applied magnetic field $(\| x)$ and the reciprocal vectors $G\{120\}$ are distributed randomly on the $y z$ plane (see Fig. 5). Because the crystal has three mutually orthogonal 2-fold axes $(a, b$, and $c$ axes), the diffractions belonging to $\{120\}$ are not distinguishable in a MOMA, neither are the diffractions $\{002\}$. With an increase in rotation speed, the $\{120\}$ diffractions on the equator disappeared and moved to locations around $\pm 45^{\circ}$ and $\pm 135^{\circ}$ when $\omega=20 \mathrm{rpm}$. At the same time, the $\{002\}$ diffractions appeared on the meridian at this rotation speed. This indicates that the $b^{*}$ axis was directed to the $z$-axis, and there was rotational symmetry about the $b^{*}$ axis. The alignment of the $\chi_{3}$ axis $\left(\| b^{*}\right)$ in the direction of the rotation axis $(\| z)$ is 


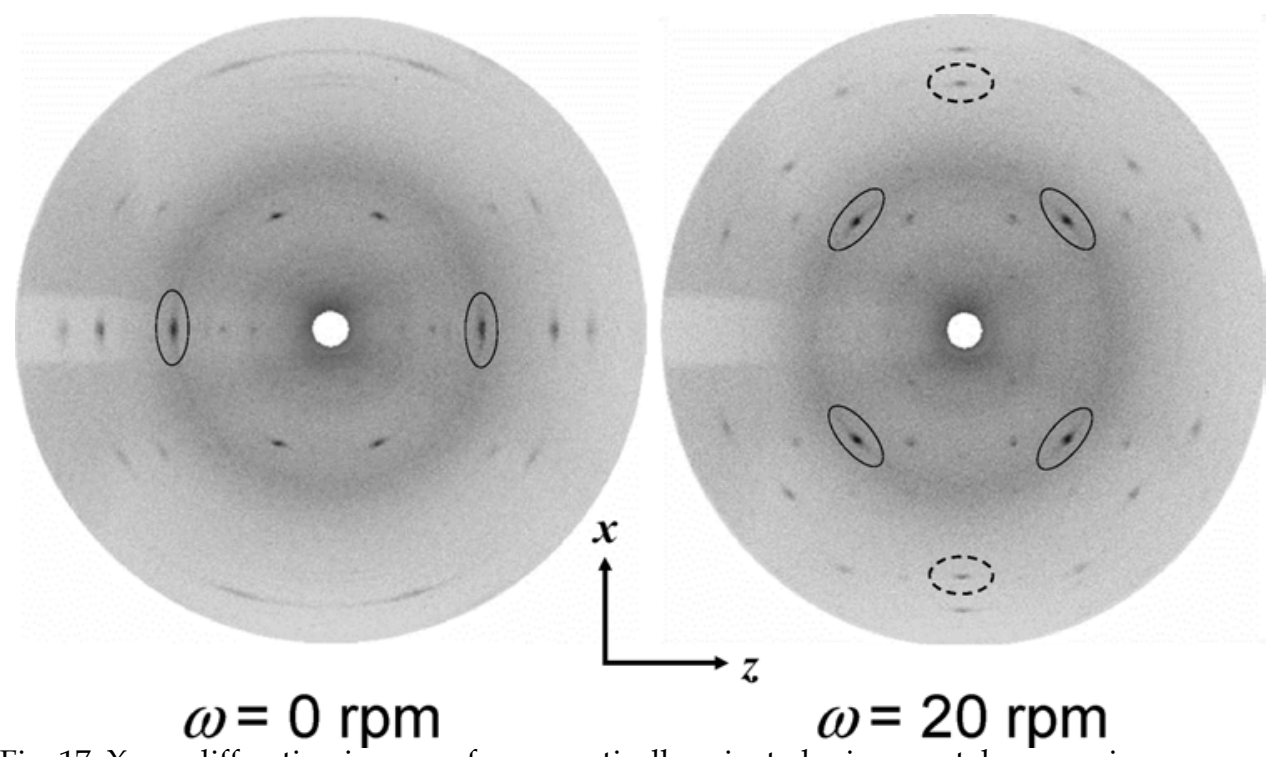

Fig. 17. X-ray diffraction images of a magnetically oriented microcrystal suspension (MOMS) obtained at $\omega=0$ and $20 \mathrm{rpm}$. Solid and broken circles indicate the $\{120\}$ and $\{002\}$ diffraction spots, respectively. (Reprint from (Matsumoto et al., 2011): Cryst. Growth Des. Vol.11(2011) pp. 945-948, No. 4, DOI: 10.1021/cg200090u. Copyright 2011 American Chemical Society)

based on the behavior of a magnetically uniaxial particle (T. Kimura, 2009; Yamaguchi et al., 2010). We successfully obtained two fiber X-ray diffraction patterns from a microcrystal suspension (crystal size of ca. 2-20 $\mu \mathrm{m}$ ) oriented in a magnetic field of $1 \mathrm{~T}$ generated by permanent magnets. Diffraction spots were sharp (ca. $2-3^{\circ}$ in half width) and well separated. The results suggest a potential use of MOMS for crystal structure analysis of solid materials that do not grow into large crystals but are obtained in the form of microcrystal suspensions.

To summarize, we have introduced a new method, 3D-MOMA (three-dimensional magnetically oriented microcrystal array) method. We use a 3D-MOMA that is a composite in which microcrystals are three-dimensionally aligned. With a 3D-MOMA, we can obtain diffraction equivalent to that from a real single crystal, successfully determining the molecular structure. With this method we can convert a suspension of biaxial microcrystals with sizes of $10 \mu \mathrm{m}$ or larger to a 3D-MOMA of centimeter sizes by using magnetic fields of $2 \mathrm{~T}$ or more. Successful examples of single crystal X-ray analyses of several crystals, including inorganic, organic, and protein crystals, by using 3D-MOMA are presented. A preliminary result of neutron diffraction of 3D-MOMA is also reported. Though the application to the neutron diffraction is preliminary, the MOMA method has high potential for the neutron diffraction method because a large size 3D-MOMA can easily be prepared. The problems to be solved for the future development of this method in the area of neutron single crystal diffraction analyses are discussed. 


\begin{tabular}{|c|c|c|c|}
\hline \multicolumn{2}{|c|}{$h k l$} & $F_{\mathrm{c}}^{2}$ & $F_{\mathrm{o}}^{2}$ \\
\hline & 0 & 12.86 & 9.50 \\
\hline & 0 & 10.42 & 8.69 \\
\hline & 1 & 8.26 & 7.89 \\
\hline & 1 & 1.02 & 1.06 \\
\hline & 1 & 0.44 & 1.43 \\
\hline & 2 & 3.55 & 5.42 \\
\hline & 2 & 77.06 & 82.74 \\
\hline & 2 & 3.96 & 3.58 \\
\hline & 2 & 5.95 & 3.62 \\
\hline & 3 & 0.26 & 0.37 \\
\hline & 3 & 1.15 & 0.89 \\
\hline & 3 & 0.00 & -0.43 \\
\hline 0 & 4 & 95.88 & 167.23 \\
\hline & 4 & 19.92 & 22.84 \\
\hline 2 & 4 & 0.00 & -0.10 \\
\hline 1 & 5 & 8.57 & 10.76 \\
\hline 2 & 5 & 1.99 & 0.97 \\
\hline 0 & 6 & 10.09 & 22.55 \\
\hline & 6 & 8.99 & 12.59 \\
\hline & 6 & 56.46 & 33.00 \\
\hline 0 & 8 & 0.42 & 1.51 \\
\hline & 0 & 13.51 & 11.27 \\
\hline 4 & 0 & 4.90 & 2.57 \\
\hline 0 & 0 & 23.59 & 18.36 \\
\hline & 0 & 23.75 & 21.53 \\
\hline 2 & 0 & 1.88 & 2.84 \\
\hline & 0 & 0.06 & -0.81 \\
\hline 2 & 0 & 2.53 & 4.15 \\
\hline & 0 & 2.48 & -0.81 \\
\hline & 0 & 14.04 & 16.37 \\
\hline & 0 & 8.01 & 7.80 \\
\hline
\end{tabular}

Table 6. Calculated and measured $F^{2}$ values for diffractions from $(h k l)$ planes for L-alanine MOMA. 


\section{References}

Asai, S.; Fujiwara, M.; Kimura, T.; Liang, Z.; Uyeda, C.; Wang, B.; Yamamoto, I.; Zhang, C. (2006). Magnetic Orientation, In Magneto-Science, M. Yamaguchi, Y. Tanimoto, (Eds), ch. 5. pp. 191-247, Tokyo: Kodansha Springer. ISSN 0933-033X

Ataka, M.; Tanaka, S. (1986). The growth of large single crystals of lysozyme. Biopolymers, Vol. 25, No. 2, (February 1986), pp. 337-350.

Blake, C. C.; Koenig, D. F.; Mair, G. A.; North, A. C.; Phillips, D. C.; Sarma, V. R. (1965). Structure of hen egg-white lysozyme. A three-dimensional Fourier synthesis at 2 Angstrom resolution, Nature, Vol. 206, (22 May 1965), pp. 757 - 761, doi:10.1038/206757a0.

Blundell, T. L.; Johnson, L. N. Protein Crystallography; Academic Press: 1976.

Finke, W. (1909), Magnetische Messungen an Platinmetallen und monoklinen Kristallen, insbesondere der Eisen-, Kobult- und Nickelsalze; von Wilhelm Fink., Ann. Phys., Vol. 336, Issue 1, (6. November 1909), PP. 149-168.

Gielen, J. C.; Wolffs, M.; Portale, G.; Bras, W.; Henze, O.; Kilbinger, A. F. M.; Feast, W. J.; Maan, J. C.; Schenning, A. P. H. J.; Christianen, P. C. M. (2009). Molecular Organization of Cylindrical Sexithiophene Aggregates Measured by X-ray Scattering and Magnetic Alignment, Langmuir Vol. 25, No. 3, (January 9, 2009), PP. 1272-1276.

Glucksman, M. J.; Hay, R. D.; Makowski, L. (1986). X-ray diffraction from magnetically oriented solutions of macromolecular assemblies, Science, Vol. 231, no. 4743, (14 March 1986), pp. 1273-1276.

Graves, b. J.; Crownther, R. L.; Chandran, C.; Rumberger, J. M.; Li, S. H.; Huang, K.S.;Presky, D. H.; Familletti, P. C.; Wolitzky, B. A.; Burns, D. K. Nature 1994, 367, 537.

Hanson, J. C., Sieker, L. C. \& Jensen, L. H. (1973). Sucrose: X-ray refinement and comparison with neutron refinement, Acta Cryst. Section B, Vol. 29, Issue 4, (April 1973), pp. 797-808.

Hariss, K. D. M.; Cheung, E. Y. (2004), How to determine structures when single crystals cannot be grown: opportunities for structure determination of molecular materials using powder diffraction data, Chem. Soc. Rev., Vol. 33, No. 8, (22 Sep 2004) pp. 526-538

Herrmann, T.; Güntert, P.; Wüthrich, K. (2002). Protein NMR Structure Determination with Automated NOE Assignment Using the New Software CANDID and the Torsion Angle Dynamics Algorithm DYANA, J. Mol. Biol. Vol. 319, No. 1, (24 May 2002), pp. 209-227.

Kachalova, G. S.; Morozov, V. N.; Morozova, T. Ya.; Myachin, E. T.; Vagin, A. A.; Strokopytov, B. V.; Nekrasov, Yu. V. (1991). Comparison of structures of dry and wet hen egg-white lysozyme molecule at $1.8 \AA$ resolution, FEBS Lett., Vol. 284, Issue 1, (17 June 1991), pp. 91-94.

Kendrew, J. C.; Bodo, G.; Dintzis, H. M.; Parrish, R. G.; Wyckoff, H.; Phillips, D. C. (1958). Xray crystallography - the first image of myoglobin, Nature, Vol. 181, (8 March, 1058), pp. 662-666. 
Kimura, F.; Kimura, T.; Matsumoto, K.; Metoki, N. (2010a). Single-Crystal Neutron Diffraction Study of Pseudo Single Crystal Prepared from Microcrystalline Powder, Crystal growth \& Design, Vol. 10, No. 1, pp. 48-51.

Kimura, F.; Kimura, T.; Oshima, W., Maeyama, W.; Aburaya, K. (2010b). X-ray diffraction study of a pseudo single crystal prepared from a crystal belonging to point group 2, J. Appl. Crystallogr., Vol. 43, pp. 151-153.

Kimura, F.; Mizutani, K.; Mikami, B.; Kimura, T. (2011). Single-Crystal X-ray Diffraction Study of a Magnetically Oriented Microcrystal Array of Lysozyme, Cryst. Growth. Des., Vol. 11, No. 1, pp. 12-15.

Kimura, T.; Yamato, M.; Koshimizu, W.; Koike, M.; Kawai, T. (2000). Magnetic Orientation of Polymer Fibers in Suspension, Langmuir, Vol. 16, No. 2, PP. 858-861.

Kimura, T.; Yoshino, M. (2005). Three-Dimensional Crystal Alignment Using a TimeDependent Elliptic Magnetic Field, Langmuir, Vol. 21, No. 11, (April 30, 2005 ), pp. 4805-4808.

Kimura, T.; Kimura, F.; Yoshino, M. (2006). Magnetic Alteration of Crystallite Alignment Converting Powder to a Pseudo Single Crystal , Langmuir, Vol. 22, No. 8, (March 9, 2006) pp. 3464-3466.

Kimura, T. (2009a). Orientation of Feeble Magnetic Particles in Dynamic Magnetic Fields, Jpn. J. Appl. Phys., Vol. 48, No. 2, (Feb. 2009), pp. 020217(1-3).

Kimura, T.; Chang, C.; Kimura, F.; Maeyama, M. (2009b). The pseudo-single-crystal method: a third approach to crystal structure determination, J. Appl. Crystallogr., Vol. 42, pp. 535-537. ISSN 0021-8898

Kohama, T.; Takeuchi, H.; Usui, M.; Akiyama, J.; Sung, M.-G.; Iwai, K.; Shigeo, A. (2007). InSitu Observation of Crystal Alignment under a Magnetic Field Using X-ray Diffraction, Mater. Trans., vol. 48, No. 11, pp. 2867-2871. ISSN 1345-9678.

Maret, G. \& Dransfeld, K. (1985). Biomolecules and Polymers in High Steady Magnetic Field, in Topics in Applied Physics, Vol. 57, F. Herlach, (Ed), ch. 4. pp. 143-204, Berlin: Springer Verlag

Margiolaki, I.; Wright, J. P. (2008). Powder crystallography on macromolecules, Acta Crystallogr. Section A, Vol. 64, Part 1, (January 2008), pp. 169-180.

Matsumoto, K.; Kimura, F.; Tsukui, S., Kimura, T. (2011). X-ray Diffraction of a Magnetically Oriented Microcrystal Suspension of L-Alanine, Crystal growth E Design, Vol. 11, (March 08, 2011), pp. 945-948.

Nagendra, H. G.; Sukumar, N.; Vijayan, M. (1998). Role of water in plasticity, stability, and action of proteins: The crystal structures of lysozyme at very low levels of hydration, Proteins, Vol. 32, Issue 2, (1 August 1998), pp. 229-240.

Niimura, N. (1999). Neutrons expand the field of structural biology, Curr. Opin. Struct. Biol., Vol. 9, Issue 5, (1 October 1999), pp. 602-608.

Nye, J. F. (1985). Paramagnetic and diamagnetic susceptibility, in Physical Properties of Crystals, ch. 3, pp. 53-67. Oxford University Press, ISBN0-19-851165-5, New York

Samulski, E. T. (1986). Magnetically Oriented Solutions, Science, Vol. 234, No. 4782, (12 December 198), p. 1424.

Sato, T.; Yamada, Y.; Saijo, S.; Hori, T.; Hirose, R.; Tanaka, N.; Sazaki, G.; Nakajima, K.; Igarashi, N.; Tanaka, M.; Matsuura, Y. (2000). Enhancement in the perfection of 
orthorhombic lysozyme crystals grown in a high magnetic field(10 T), Acta Crystallogr. Section D, vol. 56, Issue 8, (August 2000) pp. 1079-1083. ISSN 0907-4449

Sousa, M. C.; Trame, C. B.; Tsuruta, H.; Wilbanks, S. M.; Reddy, V. S.; McKay, D. B. (2000). Crystal and Solution Structures of an HslUV Protease-Chaperone Complex, Vol. 103, Issue 4, (10 November 2000), pp. 633-643

Terada, N.; Suzuki, H. S.; Suzuki, T. S.; Kitazawa, H.; Sakka, Y.; Kaneko, K.; Metoki, N. (2008). In situ neutron diffraction study of aligning of crystal orientation in diamagnetic ceramics under magnetic fields, Appl. Phys. Lett., Vol. 92, Issue 11, (17 Mar 2008), pp. 112507(1-3).

van der Beek, D.; Petukhov, A. V.; Davidson, P.; J. Ferr_e, J.; Jamet, J. P.; Wensink, H. H.; Vroege, G. J.; Bras, W.; Lekkerkerker, H. N. W. (2006). Magnetic-field-induced orientational order in the isotropic phase of hard colloidal platelets, Phys. Rev. E, Vol. 73, Issue 4, (5 April 2006), pp. 041402(1-10).

Wessels, T., Baerlocher, C. \& McCusker, L. B. (1999). Single-Crystal-Like Diffraction Data from Polycrystalline Materials, Science, Vol. 284 no. 5413, (16 April 1999), pp. 477479.

Wilson, C. C.; Myles, D.; Ghosh, M.; Johnson, L. N.; Wang, W. (2005). Neutron diffraction investigations of $\mathrm{L}$ - and D-alanine at different temperatures: the search for structural evidence for parity violation, New J. Chem., Vol. 29, (07 Sep 2005), pp. 1318-1322.

Yamaguchi, M.; Ozawa, S.; Yamamoto, I. (2010). Dynamic Behavior of Magnetic Alignment in Rotating Field for Magnetically Weak Particles, Jpn. J. Appl. Phys. Vol. 49, (5 August, 2010), pp. 080213(1-3) 


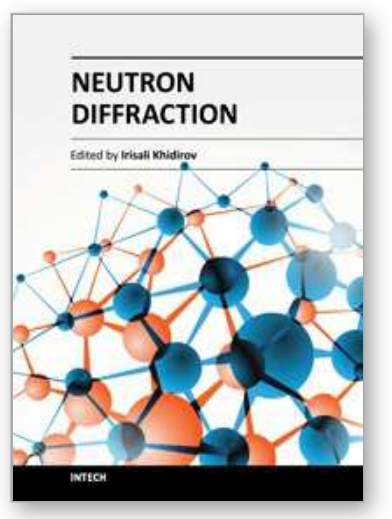

\author{
Neutron Diffraction \\ Edited by Prof. Irisali Khidirov
}

ISBN 978-953-51-0307-3

Hard cover, 286 pages

Publisher InTech

Published online 14, March, 2012

Published in print edition March, 2012

Now neutron diffraction is widely applied for the research of crystal, magnetic structure and internal stress of crystalline materials of various classes, including nanocrystalls. In the present book, we make practically short excursion to modern state of neutron diffraction researches of crystal materials of various classes. The book contains a helpful information on a modern state of neutron diffraction researches of crystals for the broad specialists interested in studying crystals and purposeful regulation of their service characteristics, since the crystal structure, basically, defines their physical and mechanical properties. Some chapters of the book have methodical character that can be useful to scientists, interested in possibilities of neutron diffraction. We hope, that results of last years presented in the book, can be a push to new ideas in studying of crystalline, magnetic structure and a macrostructure of usual crystal materials and nanocrystals. In turn, it can promote working out of new materials with new improved service characteristics and to origin of innovative ideas.

\title{
How to reference
}

In order to correctly reference this scholarly work, feel free to copy and paste the following:

T. Kimura, F. Kimura, K. Matsumoto and N. Metoki (2012). Three-Dimensional Magnetically-Oriented Microcrystal Array: A Large Sample for Neutron Diffraction Analysis, Neutron Diffraction, Prof. Irisali Khidirov (Ed.), ISBN: 978-953-51-0307-3, InTech, Available from: http://www.intechopen.com/books/neutrondiffraction/magnetically-oriented-microcrystal-array-a-large-sample-for-neutron-single-crystal-diffraction-analy

\section{INTECH}

open science | open minds

\author{
InTech Europe \\ University Campus STeP Ri \\ Slavka Krautzeka 83/A \\ 51000 Rijeka, Croatia \\ Phone: +385 (51) 770447 \\ Fax: +385 (51) 686166 \\ www.intechopen.com
}

\author{
InTech China \\ Unit 405, Office Block, Hotel Equatorial Shanghai \\ No.65, Yan An Road (West), Shanghai, 200040, China \\ 中国上海市延安西路65号上海国际贵都大饭店办公楼 405 单元 \\ Phone: +86-21-62489820 \\ Fax: $+86-21-62489821$
}


(C) 2012 The Author(s). Licensee IntechOpen. This is an open access article distributed under the terms of the Creative Commons Attribution 3.0 License, which permits unrestricted use, distribution, and reproduction in any medium, provided the original work is properly cited. 